Pérez Fernández, O. A. (2021). Modelos de localización asignación utilizando redes dinámicas a partir de ficheros GTFS y datos de TomTom. GeoFocus, Revista Internacional de Ciencia y Tecnología de la Información Geográfica (Artículos), 28, 59-81. http://dx.doi.org/10.21138/GF.751

\title{
MODELOS DE LOCALIZACIÓN ASIGNACIÓN UTILIZANDO REDES DINÁMICAS A PARTIR DE FICHEROS GTFS Y DATOS DE TOMTOM
}

\author{
ONEL ANTONIO PEREZ FERNÁNDEZ \\ Departamento de Geografía, Universidad de Panamá \\ Ciudad de Panamá 0824, Panamá \\ oneperez@ucm.es
}

\begin{abstract}
RESUMEN
Los Modelos de Localización Asignación (MLA) en Sistemas de Información Geográfica se utilizan para buscar los mejores sitios donde ubicar las instalaciones de servicios o equipamientos. Tradicionalmente, los MLA han sido aplicados sin integrar variables temporales en la red de transporte. El objetivo de este trabajo es el planteamiento de MLA incorporando el dinamismo en la red y aplicado a los hospitales públicos. Para ello se utilizan archivos de redes de transporte público dinámicas (GTFS) y redes de carreteras con datos históricos de tráfico (en este caso de la empresa TomTom), lo que permite evaluar el efecto de los cambios en las frecuencias del transporte público y la congestión del tráfico en los resultados de los modelos. Los resultados muestran los impactos en la cantidad de demanda cubierta, considerando el comportamiento temporal de la red a lo largo del día.
\end{abstract}

Palabras clave: Modelos de Localización Asignación; SIG; GTFS; TomTom; Big Data.

LOCATION ALLOCATION MODELS USING DYNAMIC NETWORKS FROM GTFS FILES AND TOMTOM DATA

\section{ABSTRACT}

Location Allocation Models (LAM) in Geographic Information Systems are used to find the best sites for locating service or equipment facilities. Traditionally, LAMs have been applied without integrating temporal variables in the transportation network. The objective of this work is the LAM approach, incorporating dynamism in the network and applied to public hospitals. For this purpose, dynamic public transport network files $(G T F S)$ and road networks with historical traffic data (in this case from the TomTom company) are used; this allows evaluating the effect of changes in public transport frequencies and traffic congestion on the model results. The results show the impacts on the amount of demand covered, considering the temporal behaviour of the network throughout the day. 


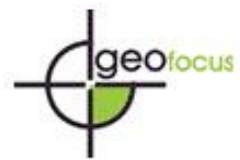

Pérez Fernández, O. A. (2021). Modelos de localización asignación utilizando redes dinámicas a partir de ficheros GTFS y datos de TomTom. GeoFocus, Revista Internacional de Ciencia y Tecnología de la Información Geográfica (Artículos), 28, 59-81. http://dx.doi.org/10.21138/GF.751

Keywords: Location Allocation Models; GIS; GTFS; TomTom; Big Data

\section{Introducción}

Los modelos de Localización Asignación (MLA) son utilizados para resolver una amplia variedad de problemas del sector público y privado (Brandeau y Chiu 1989). Por ejemplo, se han empleado para encontrar la mejor ubicación de instalaciones de salud (Ajebon y Asikhia, 2013; Chukwusa y Comber, 2018; Özceylan et al. 2017), instalaciones para el tratamiento de desechos sólidos (Alonso 2016, El-Hallaq y Mosabeh, 2019; Erfani et al. 2018), para estaciones de bicicletas (García-Palomares et al. 2012), escuelas (Wondwossen y Bedasa, 2019) y muchas otras aplicaciones. Tradicionalmente, los MLA se abordaban considerando la red de forma estática, ya que no existían datos que permitieran conocer las condiciones del tráfico o la frecuencia del transporte público. Hoy en día, con la aparición de nuevas fuentes de datos (como Big Data), es posible incorporar dinamismo a la red, lo que permite simular escenarios más realistas.

La información sobre las redes de transporte ha mejorado gracias a la gran cantidad de datos generados por las empresas de navegación como TomTom, y la disponibilidad de archivos de transporte público GTFS (General Transit Feed Specification) gestionada por las autoridades de transporte (Moya-Gómez et al. 2018). Los registros almacenados por ambas redes han hecho posible enriquecer los ejes de las calles con datos históricos del tráfico del transporte privado y las frecuencias del transporte público, esto permite evaluar el impacto en la movilidad de la demanda en un momento determinado del día. En efecto, el Big Data ofrece una variedad de oportunidades para el análisis dinámico de la accesibilidad a los servicios públicos o privados. En los últimos años, se han desarrollado algunos trabajos que incorporan Big Data a los MLA, específicamente para el análisis de la demanda o de la oferta (Kotavaara 2018, Park y Young Sohn 2017, Tu et al. 2016, Yang et al. 2020, Zhang et al. 2019, Zhou et al. 2020). Sin embargo, aparecen pocos estudios que utilicen archivos GTFS o datos de TomTom para el planteamiento de los modelos de localización asignación.

Esta investigación aporta un nuevo enfoque a los MLA, al simular el dinamismo de la red de transporte público y privado. De este modo, se pueden realizar planteamientos que incluyan el elemento temporal en el funcionamiento de las redes. Lo que permite la evaluación de la demanda asignada a los hospitales actuales y la ubicación óptima de nuevos centros hospitalarios. Por lo tanto, esta investigación tiene doble propósito. En primer lugar, plantear modelos de localización asignación, incorporando el dinamismo en el funcionamiento tanto de la red de carreteras como del transporte público, para evaluar el impacto en la cobertura de la demanda de los hospitales actuales de Madrid, producto de los cambios en las frecuencias del transporte público y la congestión en el transporte privado. En segundo lugar, analizar la ubicación óptima de un nuevo hospital considerando el comportamiento durante el día de la red del transporte público y privado. Para esto utilizamos archivos GTFS y datos históricos de tráfico de TomTom. Ambos conjuntos de datos permitieron la construcción de las redes que simularon el desplazamiento en transporte público y en coches privados.

Los principales hallazgos de este trabajo demuestran que la demanda captada por los equipamientos de salud se ve afectada por los cambios en las condiciones de la red de transporte en un determinado periodo del día. Cuando se agrega un nuevo hospital mejora la cobertura del servicio actual. Además, la ubicación del nuevo hospital cambia de acuerdo con el comportamiento diario de la red. Los resultados tienen aplicaciones prácticas para las autoridades 


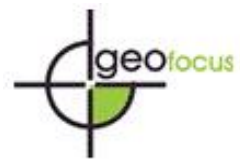

Pérez Fernández, O. A. (2021). Modelos de localización asignación utilizando redes dinámicas a partir de ficheros GTFS y datos de TomTom. GeoFocus, Revista Internacional de Ciencia y Tecnología de la Información Geográfica (Artículos), 28, 59-81. http://dx.doi.org/10.21138/GF.751

locales y las empresas que se ven afectadas por la variación en las frecuencias del transporte público y por la congestión en el transporte privado.

Este artículo está estructurado de la siguiente manera: después de la introducción, la sección 2, describe la literatura revisada. La sección 3, presenta el área de estudio y los datos, en la sección 4 , se explica la metodología utilizada y los resultados obtenidos se presentan en la sección 5 y en la sección 6 , se establece una serie de conclusiones.

\section{Revisión de literatura}

\subsection{El uso de redes dinámicas de transporte público: GTFS}

Los ficheros GTFS incluyen todo lo relativo a las rutas, paradas, horarios, frecuencias de viajes y otros datos de interés concernientes a las rutas de transporte público en una ciudad. A través de estos archivos se pueden implementar una diversidad de aplicaciones para ofrecer información de interés en la planificación del viaje, la creación de horarios, la accesibilidad, las herramientas necesarias para la planificación en tiempo real y los sistemas de voz interactiva (Antrim y Barbeau, 2013).

En los últimos años, los archivos GTFS se han utilizado en el mundo académico para realizar investigaciones relacionadas con la movilidad y la accesibilidad a los centros de servicios. Así tenemos que Farber et al. (2014) realizaron un análisis de la accesibilidad por medio de matrices de origen destino, desde las secciones censales a los supermercados. Calculan el tiempo de viaje por medio del transporte público, para ello seleccionan un día de la semana en particular. Presentan resultados sobre las diferencias en el nivel de acceso a los supermercados según los grupos sociales y la edad en dos ciudades de Estados Unidos. Otro caso lo encontramos en una investigación cuyo objetivo era determinar la movilidad de los usuarios del sistema de transporte, apoyándose en la información proveniente de los archivos GTFS. Además, utilizan los registros del abordaje y el conteo de pasajeros dentro del bus, tomando como muestra del estudio un día de la semana en varias franjas horarias (Nassir et al., 2012). Al respecto, el trabajo de Stępniak y Goliszek (2017) utiliza archivos GTFS para analizar el nivel de accesibilidad durante diversas horas del día. Incorporando la componente temporal a este tipo de estudio para obtener resultados que permiten apreciar, en mapas y gráficas, el nivel de equidad a las distintas secciones censales, apoyándose en indicadores como el índice de Gini, la desviación estándar, entre otros.

Por otro lado, existen pocos trabajos que utilicen los archivos GTFS en el planteamiento de los modelos de localización asignación. Un ejemplo, es el trabajo de Kotavaara (2018) donde emplea datos GTFS con el fin de encontrar lugares óptimos para los servicios de atención primaria en Finlandia. En su planteamiento utiliza la solución minimizar impedancia, estimando el tiempo mínimo desde los puntos de demanda hacia los hospitales. Luego de la revisión de la literatura se ha encontrado que el principal enfoque de los estudios con datos GTFS, es la elaboración de medidas de accesibilidad a servicios y al trabajo (Karner 2018, Slovic et al. 2019, Stępniak et al. 2019), el desarrollo de herramientas para planificar viajes y de visualización de los datos (Fayyaz S. et al. 2017, Jakimavičius et al. 2019).

\subsection{Uso de datos de redes dinámicas de transporte privado: TomTom}

La oferta de nuevas redes de transporte se ha incrementado con la disponibilidad de redes de empresas como TomTom, HERE, Inrix o el proyecto colaborativo de OSM entre otros, representando novedosas fuentes de datos, que abren nuevas oportunidades para realizar 


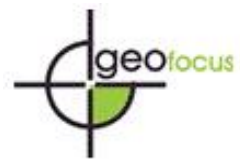

Pérez Fernández, O. A. (2021). Modelos de localización asignación utilizando redes dinámicas a partir de ficheros GTFS y datos de TomTom. GeoFocus, Revista Internacional de Ciencia y Tecnología de la Información Geográfica (Artículos), 28, 59-81. http://dx.doi.org/10.21138/GF.751

investigaciones innovadoras que buscan incorporar en sus estudios variables espaciales y temporales de gran nivel de resolución (Moya-Gómez et al. 2018).

TomTom es una empresa especializada en tecnología de localización, puesto que desarrolla productos cartográficos, programas de navegación, de congestión vehicular histórica y en tiempo real. Ellos administran grandes volúmenes de datos capturando y almacenando registros de tráfico y de velocidad. Además, proporcionan información detallada de los niveles de flujo sobre las autovías en las principales ciudades del mundo. Las investigaciones basadas en el uso de datos TomTom son escasas en la literatura académica. Los estudios encontrados abordan temáticas relacionadas con la accesibilidad. Un ejemplo es el trabajo propuesto por Pritchard et al. (2019) donde examinan el potencial de la bicicleta como medio para reducir las desigualdades en la accesibilidad espacio-temporal en los viajes hacia el empleo. Para lograr su objetivo utilizaron los perfiles de velocidad de TomTom en la elaboración de los modelos de accesibilidad y comparan los resultados frente a datos de archivos GTFS. Por su parte, Tanveer et al. (2020) se enfocó en estudiar patrones de circulación, realizando monitoreos de las variaciones del flujo en las ciudades; utilizó los índices de tráfico de TomTom en la validación de su estudio.

En la publicación realizada por Dingil et al. (2018) se emplearon modelos de regresión utilizando los niveles de congestión de TomTom, para comparar indicadores de transporte en diferentes áreas urbanas y valorar su grado de accesibilidad. Por otro lado, García-Palomares et al. (2018) proponen un análisis de la accesibilidad urbana, teniendo en cuenta el rendimiento de la red y el atractivo de los destinos mediante un enfoque dinámico, donde se aprovechan los datos de TomTom y Twitter. En otro trabajo, Moya-Gómez y García-Palomares (2017) examinan los cambios en la accesibilidad de los automóviles, en el transcurso del día, como consecuencia del embotellamiento de la red de carreteras en algunas ciudades de Europa, para esto se aplicaron modelos de accesibilidad. Por su parte, Moyano et al. (2018) analizan los tiempos de ingreso y egreso a las estaciones de trenes de alta velocidad entre dos ciudades españolas, utilizando datos de TomTom y Twitter.

Recogiendo lo más importante, los autores analizados se centran, sobre todo, en cuestiones relacionadas con los efectos de la congestión vehicular en las ciudades y la influencia en el nivel de accesibilidad; utilizando los productos que ofrece la empresa TomTom como, por ejemplo, los perfiles históricos de velocidad y los índices de congestión, para el planteamiento de los modelos desarrollados. Tras la revisión de literatura, no se han encontrado estudios de MLA utilizando datos de TomTom en la confección de la red.

\section{3. Área de estudio y datos}

\section{1 Área de estudio}

El área seleccionada para el estudio es el municipio de Madrid. Administrativamente está formado por 21 distritos. Es considerada una de las principales metrópolis europeas. La diversidad de atracciones turísticas e instalaciones de servicio y ocio atraen millones de visitantes nacionales y extranjeros todos los años. De acuerdo con el Padrón Municipal de Habitantes 2019, el municipio de Madrid contaba con 3266126 habitantes (50 \% de la población de la Comunidad de Madrid) que cubren una superficie de 60 445,52 ha con una densidad de población aproximada de 55 habitantes por hectárea. Los servicios de salud dentro del municipio lo forman 129 centros de salud, 35 hospitales y centros de atención de salud mental distribuidos en siete áreas de salud. Este trabajo se enfoca en los hospitales públicos y las áreas de salud existentes dentro de la zona objeto de estudio (Figura 1). 


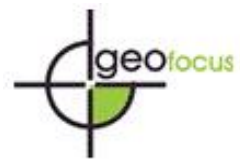

Pérez Fernández, O. A. (2021). Modelos de localización asignación utilizando redes dinámicas a partir de ficheros GTFS y datos de TomTom. GeoFocus, Revista Internacional de Ciencia y Tecnología de la Información Geográfica (Artículos), 28, 59-81. http://dx.doi.org/10.21138/GF.751

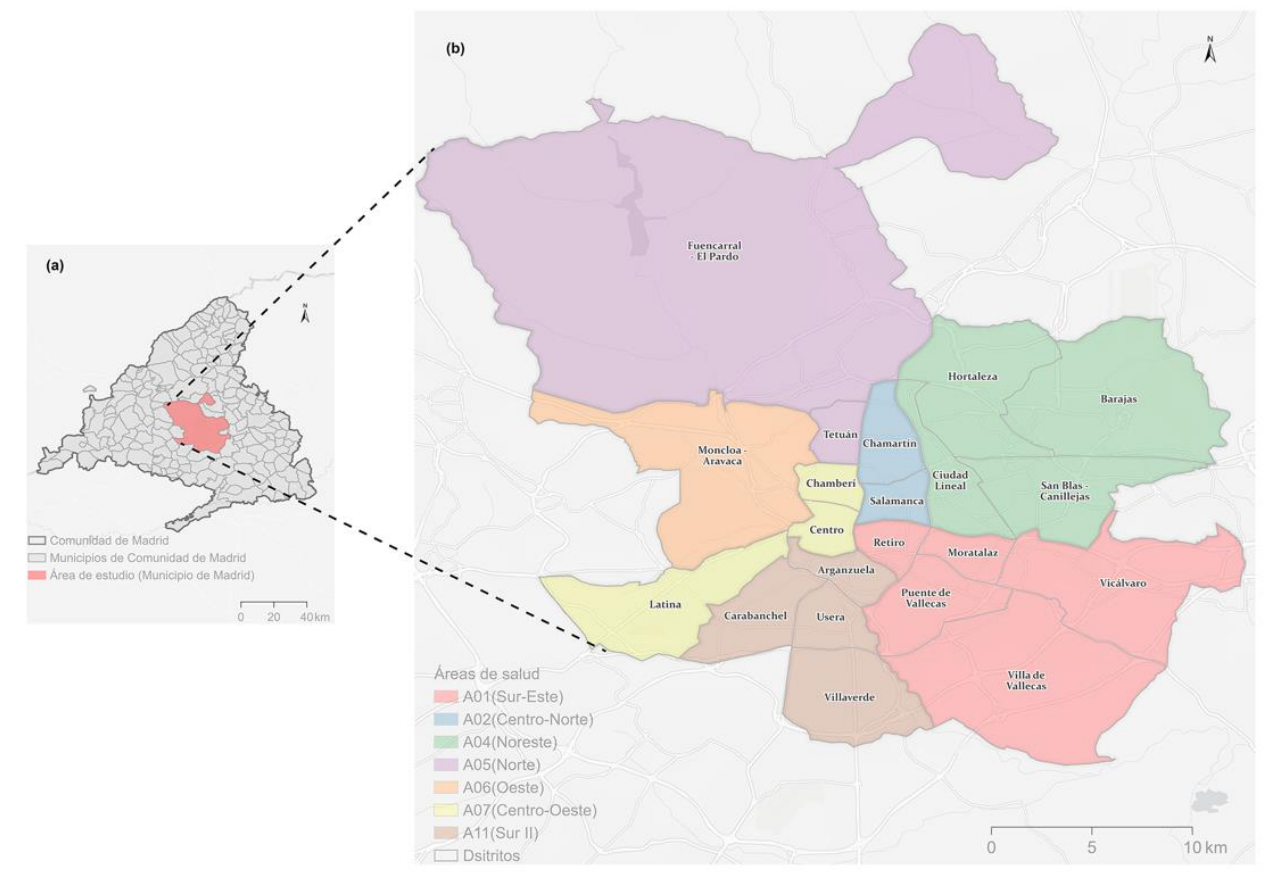

Figura 1. Área de estudio. (a) Comunidad de Madrid dividida en municipios y el área de estudio. (b) Áreas de salud dentro del área de estudio.

Fte. Elaboración propia.

\subsection{Datos}

\subsubsection{Hospitales}

Los MLA requieren de puntos candidatos a los cuales asignarles la demanda a cubrir. Para este fin, se utilizó una capa de hospitales públicos (geometría de puntos) del municipio de Madrid y los hospitales asignados por áreas de salud. Ambas capas fueron obtenidas del servidor de datos espaciales de la Comunidad de Madrid. En el 2020 el municipio de Madrid tenía 35 hospitales públicos y siete áreas de salud. En el caso del modelo que asigna un nuevo hospital se emplean los hospitales de las áreas de salud actuales como ubicaciones requeridas, mientras que los candidatos se obtienen a partir de los centroides de las secciones censales.

\subsubsection{Secciones censales y población}

Para obtener la demanda a cubrir, se empleó las secciones censales descargadas del servidor de datos de la Comunidad de Madrid. A éstas se les agrega un campo con la población total, según el padrón continuo de población para el año 2019, disponible en el servidor del Instituto Nacional de Estadística (INE 2020).

\subsubsection{Red de transporte público}

Para esta información se utilizó la red de calles descargada del servidor de $O S M$, con el fin de simular el desplazamiento de la población en transporte público, esta red fue enriquecida con datos de los archivos GTFS del año 2018 descargados de la página web del Consorcio Regional 
Pérez Fernández, O. A. (2021). Modelos de localización asignación utilizando redes dinámicas a partir de ficheros GTFS y datos de TomTom. GeoFocus, Revista Internacional de Ciencia y Tecnología de la Información Geográfica (Artículos), 28, 59-81. http://dx.doi.org/10.21138/GF.751

de Transportes de Madrid (https://datos.crtm.es/). Los ficheros GTFS se componen de una colección de archivos en formato de texto que reúnen datos relacionados con el transporte público de Madrid. Mantienen registros de paradas, rutas, viajes, calendario, frecuencias y agencia que ofrece el servicio.

\subsubsection{Red de transporte privado}

Esta red se creó con datos de tráfico histórico obtenidos de la empresa TomTom, con la finalidad de simular la movilidad de la población en el transporte privado. Dichos datos cuentan con información de congestión en la ciudad de Madrid para el año 2018.

\section{Metodología}

Se tomaron en cuenta tres etapas para realizar el planteamiento de los modelos de localización. En primer lugar, se requiere tener sitios candidatos y sitios que representen la demanda. Luego, para lograr la conectividad de estos sitios se necesitó la creación de una red. Por último, se implementó una de las soluciones que ofrece el programa ArcMap 10.6.

\subsection{Candidatos y Demanda potencial}

Se eligió la red de hospitales públicos como sitios candidatos para la ejecución del modelo de localización asignación. Además, se realizó un modelo utilizando como candidatos los hospitales asignados a las áreas de salud que ofrecen el servicio médico a los habitantes del municipio de Madrid. A efectos de gestión de la sanidad, la Comunidad de Madrid se organiza en áreas de salud. Se trata del área geográfica de zonificación sanitaria que agrupa los recursos de atención primaria y especializada, teniendo en cuenta factores geográficos, culturales, epidemiológicos y las infraestructuras existentes. Las áreas están conformadas por la agregación de varios distritos.

Por otro lado, los puntos de demanda se tomaron a partir de los centroides de las secciones censales, las cuales contienen un campo que incluye la población que se utilizó para ponderar la cantidad de habitantes que cubrirá cada hospital. Además, se ejecutó un modelo de localización asignación que incluye un nuevo hospital, utilizando los hospitales actuales como puntos requeridos, mientras que los candidatos se obtienen de los centroides de las secciones censales.

La Figura 2, muestra los hospitales y los puntos de calor donde se concentra la demanda que será asignada a los hospitales. El color amarillo representa las zonas con densidades de población más altas. Se evidencia una concentración de la población en la zona central del área estudiada. Marcando valores altos las áreas de salud: Centro-Norte, Sur II y Centro-Oeste. 


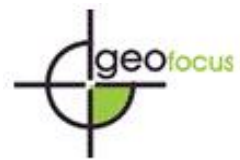

Pérez Fernández, O. A. (2021). Modelos de localización asignación utilizando redes dinámicas a partir de ficheros GTFS y datos de TomTom. GeoFocus, Revista Internacional de Ciencia y Tecnología de la Información Geográfica (Artículos), 28, 59-81. http://dx.doi.org/10.21138/GF.751

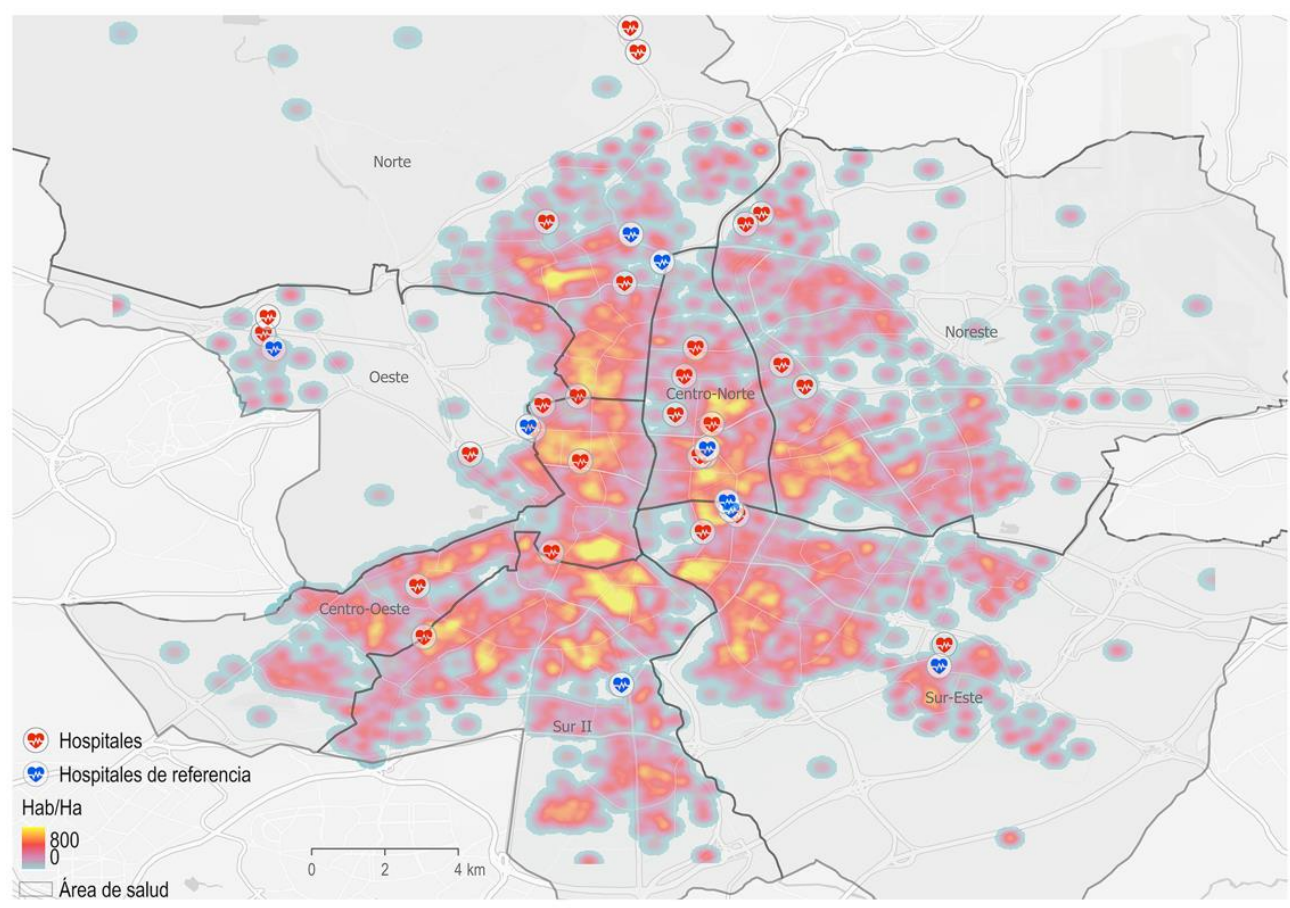

Figura 2. Distribución de hospitales y demanda.

Fte. Elaboración propia.

4.2 Redes de carreteras para el transporte público y privado

Para incorporar el dinamismo en las calles se construyeron dos redes que simulan el desplazamiento de la población en transporte público y en transporte privado. La red de transporte público se confeccionó a partir de los datos GTFS. Estos ficheros contienen información relacionada a las frecuencias de viajes, tiempo, calendario del servicio de bus urbano (EMT), metro, cercanías y autobuses interurbanos. Para construir la red se emplearon las herramientas DisplayGTFS y Network Analyst del programa ArcMap 10.6 de la casa comercial ESRI (Environmental Systems Research Institute). Como atributo de impedancia se estableció el tiempo de viaje en transporte.

Por otro lado, la red que simula el desplazamiento en transporte privado se diseñó con la extensión Network Analyst, utilizando los datos de la empresa TomTom, específicamente, el producto perfiles de velocidad, donde registra el tiempo promedio de los viajes efectuados por los usuarios de los navegadores TomTom. Dicha empresa almacena los registros históricos de velocidad, con los cuales se obtuvo perfiles de tráfico en los diferentes ejes que componen la red de calles. Además, se crearon tablas que contienen información de los perfiles de velocidad por hora y día.

En los mapas de la Figura 3, se muestra la frecuencia de viajes, en el transporte público, los jueves. Se observó que las horas 8:00 y 18:00 tienen mayor frecuencia de viaje por hora. En cambio, en las horas 10:00 y 20:00 disminuye la frecuencia de viajes. Por otro lado, la Figura 4, muestra el nivel de tráfico en la red utilizando los datos de perfiles históricos de velocidad de la empresa TomTom. Se observó que la fluidez del tráfico es menor, sobre todo, en las horas 8:00 y 


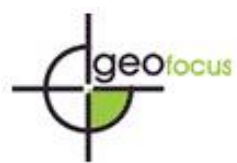

Pérez Fernández, O. A. (2021). Modelos de localización asignación utilizando redes dinámicas a partir de ficheros GTFS y datos de TomTom. GeoFocus, Revista Internacional de Ciencia y Tecnología de la Información Geográfica (Artículos), 28, 59-81. http://dx.doi.org/10.21138/GF.751
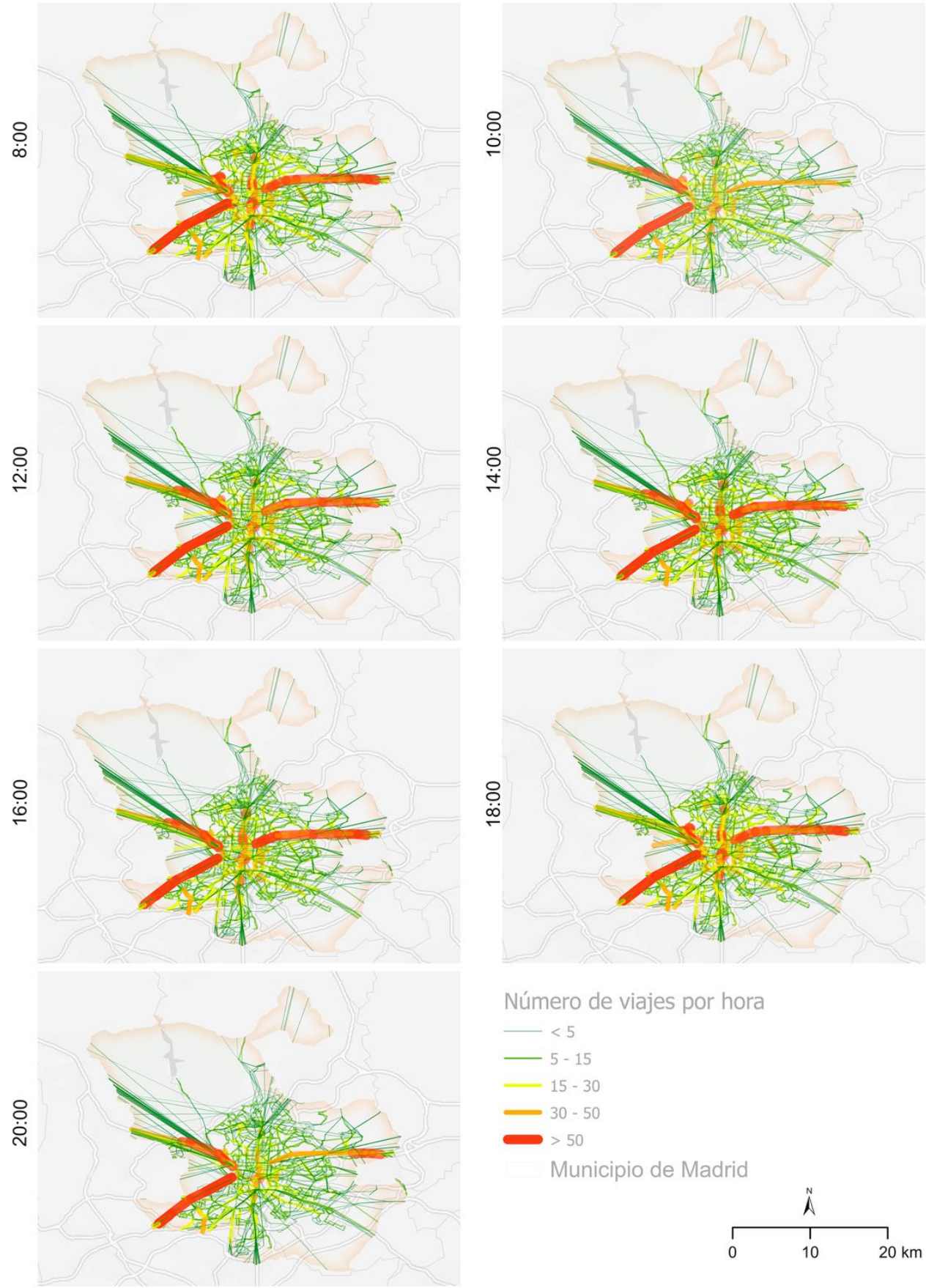

Número de viajes por hora

$-<5$

$-5-15$

$-15-30$

$-30-50$

$>50$

Municipio de Madrid

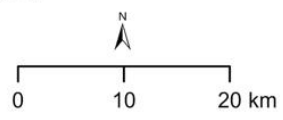

Figura 3. Frecuencia de viaje en transporte público los jueves (GTFS). Fte. Elaboración propia. 
Pérez Fernández, O. A. (2021). Modelos de localización asignación utilizando redes dinámicas a partir de ficheros GTFS y datos de TomTom. GeoFocus, Revista Internacional de Ciencia y Tecnología de la Información Geográfica (Artículos), 28, 59-81. http://dx.doi.org/10.21138/GF.751
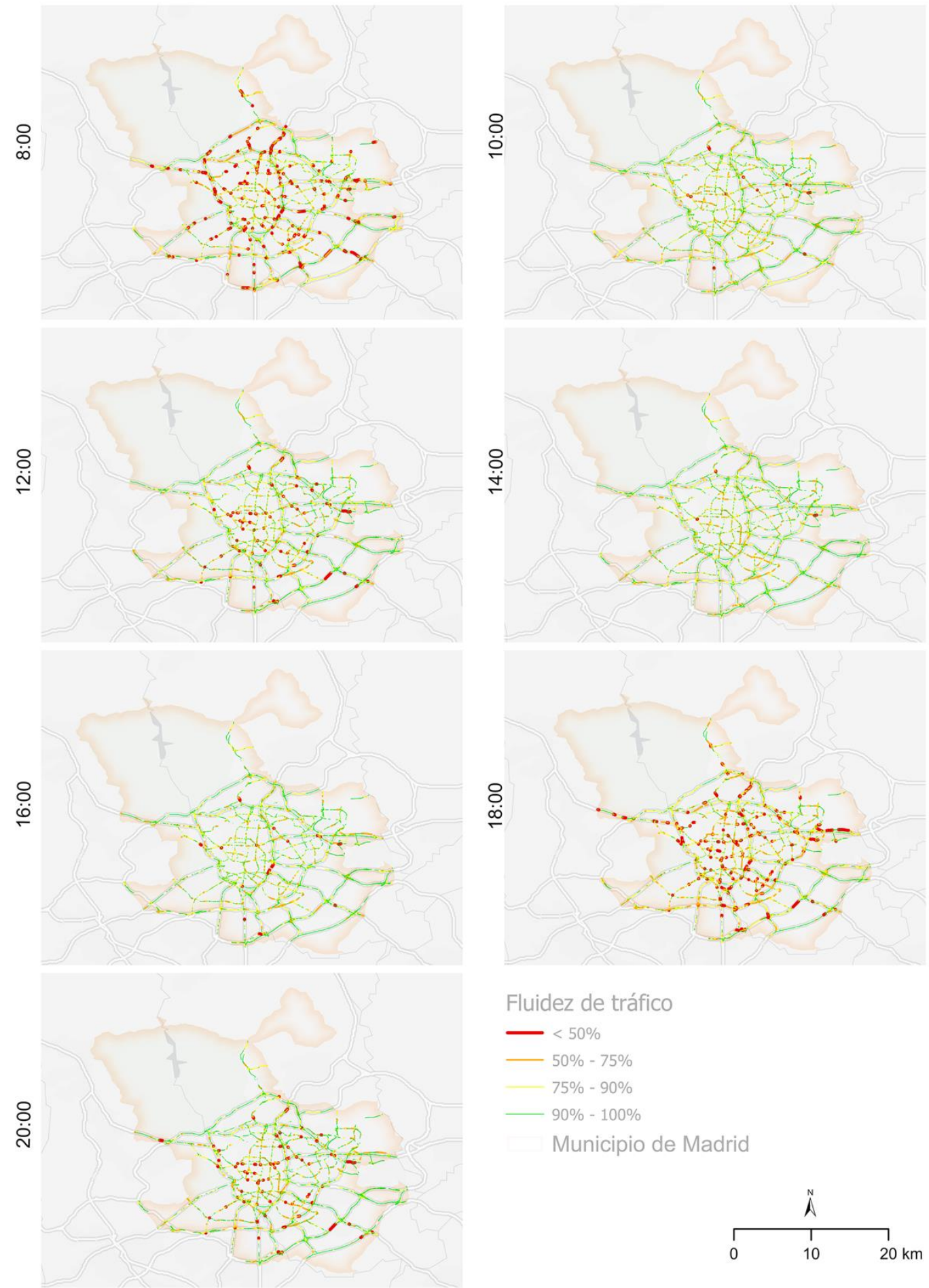

Fluidez de tráfico

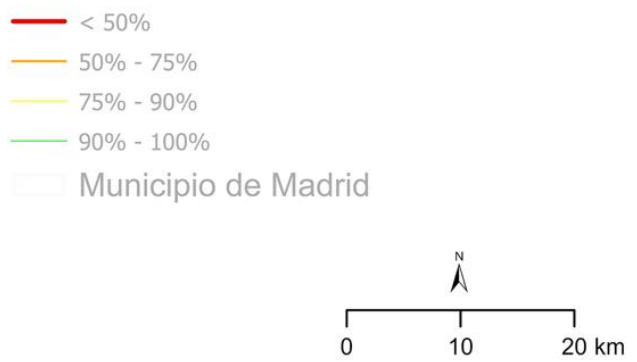

Figura 4. Fluidez del tráfico los jueves (TomTom).

Fte. Elaboración propia.

18:00. El resto de las franjas horarias no presentaron cambios significativos en los distintos ejes de calles. 


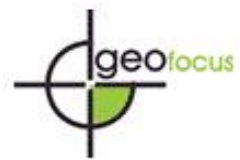

Pérez Fernández, O. A. (2021). Modelos de localización asignación utilizando redes dinámicas a partir de ficheros GTFS y datos de TomTom. GeoFocus, Revista Internacional de Ciencia y Tecnología de la Información Geográfica (Artículos), 28, 59-81. http://dx.doi.org/10.21138/GF.751

\subsection{Modelos de localización asignación}

Con la red de transporte público y privado construida se procedió a ejecutar los modelos de localización asignación, utilizando el módulo Location Allocation de Network Analyst de ArcMap 10.6 (ESRI) con la finalidad de evaluar en qué momento del día los hospitales captan mayor cobertura de población. Se seleccionó la solución de maximizar la atención. Dicha solución plantea que las instalaciones se eligen asignando el máximo peso de demanda posible a los equipamientos, asumiendo que el peso de la demanda disminuye en relación con la distancia entre los sitios candidatos y los puntos de demanda potencial. Según Alonso (2016) se maximiza la demanda localizada dentro de un umbral de distancia, dando más importancia a la demanda cercana a los puntos de oferta. Maximizar la asistencia supone que cuando más lejos tengan que desplazarse las personas para hacer uso de un equipamiento, la probabilidad de que sea utilizada es menor (Erfani et al. 2018, Rahman et al. 2021).También, se consideró un umbral de 30 minutos de viaje. Se plantearon escenarios para dos días de la semana (específicamente los jueves y los sábados). Los modelos se ejecutaron con la red GTFS (transporte público) y la red de TomTom (transporte privado) en diferentes franjas horarias: 8:00, 10:00, 12:00, 14:00, 16:00, 18:00 y 20:00; con el propósito de valorar el efecto de la fluctuación en las frecuencias del transporte público y de los niveles de congestión de la red de transporte privado sobre la cantidad de demanda atendida.

Además, se ejecutó otro MLA utilizando como candidatos solamente los hospitales de referencia que cubren las áreas de salud del municipio de Madrid, el resto de los parámetros del modelo son los mismos que se aplicaron a toda la red de hospitales (Figura 5). Por último, se propuso un modelo de localización asignación que incluyó un nuevo hospital. Como instalaciones requeridas se empleó la ubicación de los hospitales actuales, mientras que los candidatos procedían de los centroides extraídos de las secciones censales. Los otros parámetros fueron similares a los propuestos en los modelos anteriores.

\section{Resultados}

\subsection{Demanda cubierta por la red de hospitales}

Se calculó la demanda cubierta por los hospitales a diferentes horas del día con ambas redes. Los resultados (Tabla 1 y la Figura 6) indican el cambio en la demanda asignada a los hospitales en el transcurso del día utilizando la red GTFS. El jueves a las 12:00 se registra el cambio porcentual más bajo. Mientras que, a las 8:00 de la mañana se cubre mayor porcentaje de la población, esto se debe a la frecuencia más alta del transporte público a esa hora. El comportamiento de la demanda el sábado es lo contrario, registrando a las 8:00 la menor población cubierta y el incremento a las 14:00 y 20:00 horas. En general, los jueves tienen una demanda cubierta superior al sábado. Este comportamiento es producto de las frecuencias de transporte público más altas en las primeras horas de la mañana de los días laborables (jueves) y bajas en las horas valle y el fin de semana. 


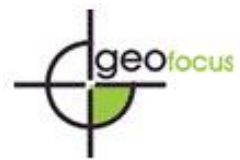

Pérez Fernández, O. A. (2021). Modelos de localización asignación utilizando redes dinámicas a partir de ficheros GTFS y datos de TomTom. GeoFocus, Revista Internacional de Ciencia y Tecnología de la Información Geográfica (Artículos), 28, 59-81. http://dx.doi.org/10.21138/GF.751

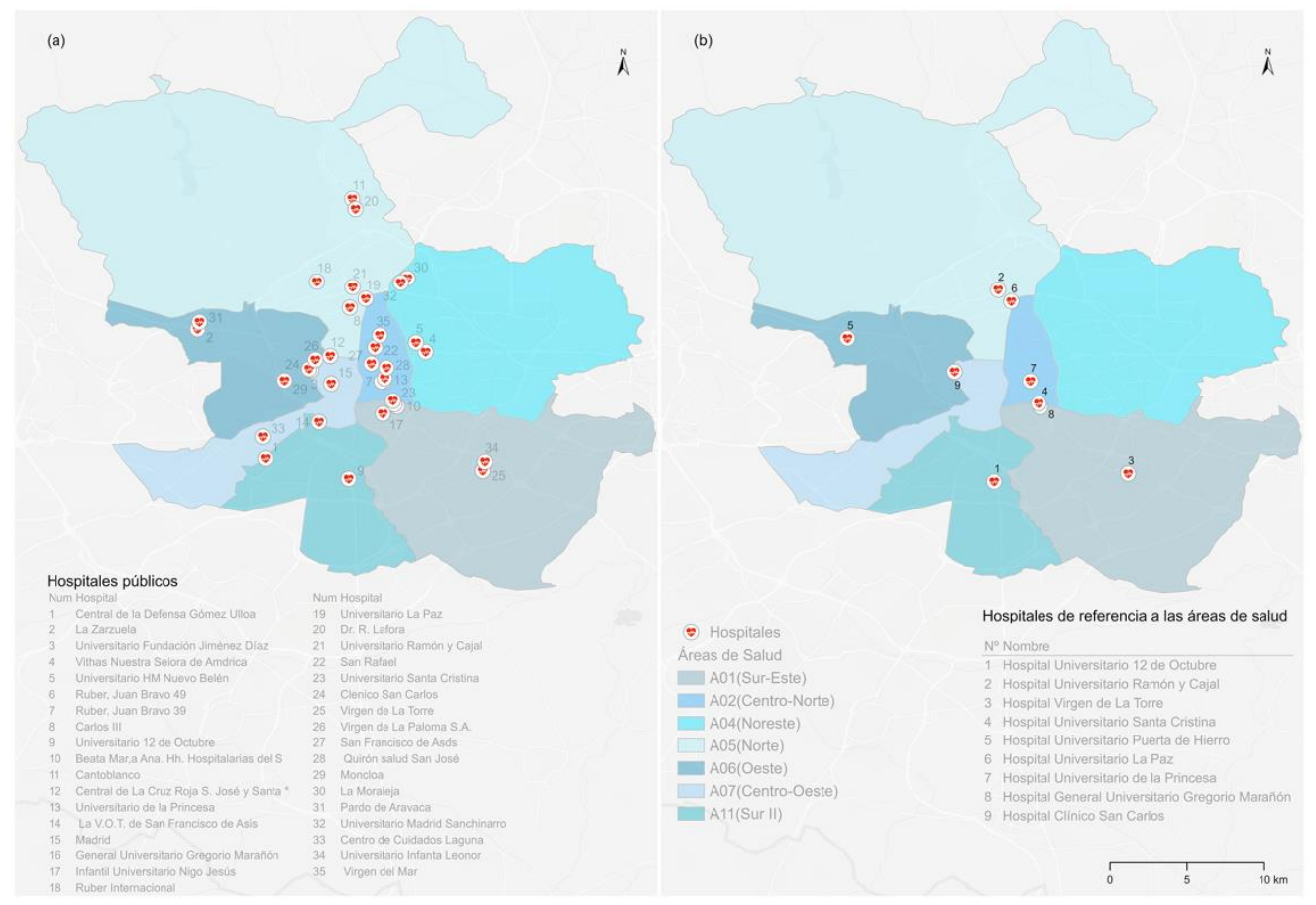

Figura 5. Red de hospitales y los hospitales de referencia asignados a las áreas de salud.

Fte. Elaboración propia.

Los resultados obtenidos utilizando la red TomTom (Tabla 2), reflejan una mayor cobertura en el porcentaje de población, con registros que presentan variaciones entre $75 \%$ y $80 \%$. El análisis de la Figura 7, resalta que el sábado se logra cubrir una mayor demanda que el jueves, debido a la menor congestión vial. Al estudiar el comportamiento de la demanda en las distintas franjas horarias, notamos que las horas donde se presentan los valores mínimos son las 8:00 y las 18:00, los jueves con momentos de mayor congestión. Se produce una situación contraria al transporte público, mejores accesos en las horas valle y el fin de semana.

Tabla 1. Demanda cubierta por hospitales utilizando la red GTFS

\begin{tabular}{ccccccccc}
\hline \multirow{3}{*}{ Horas } & \multicolumn{3}{c}{ Jueves 30 minutos } & \multicolumn{5}{c}{ Sábado 30 minutos } \\
\cline { 2 - 8 } & Total & $\%$ & Cambio & Cambio\% & Total & $\%$ & Cambio & Cambio\% \\
\hline 08:00 & 1469888 & 45,6 & & & 1371781 & 42,6 & & \\
$10: 00$ & 1430688 & 44,4 & -39200 & $-2,7$ & 1383042 & 42,9 & 11261 & 0,8 \\
$12: 00$ & 1419672 & 44,0 & -50216 & $-3,4$ & 1401828 & 43,5 & 30047 & 2,2 \\
$14: 00$ & 1438650 & 44,6 & -31238 & $-2,1$ & 1413210 & 43,8 & 41429 & 3,0 \\
$16: 00$ & 1437526 & 44,6 & -32362 & $-2,2$ & 1400802 & 43,5 & 29021 & 2,1 \\
$18: 00$ & 1446992 & 44,9 & -22896 & $-1,6$ & 1405833 & 43,6 & 34052 & 2,5 \\
$20: 00$ & 1430474 & 44,4 & -39414 & $-2,7$ & 1416392 & 43,9 & 44611 & 3,3 \\
\hline
\end{tabular}

Fte. Elaboración propia. 


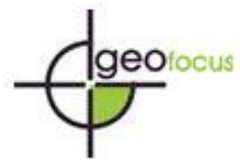

Pérez Fernández, O. A. (2021). Modelos de localización asignación utilizando redes dinámicas a partir de ficheros GTFS y datos de TomTom. GeoFocus, Revista Internacional de Ciencia y Tecnología de la Información Geográfica (Artículos), 28, 59-81. http://dx.doi.org/10.21138/GF.751

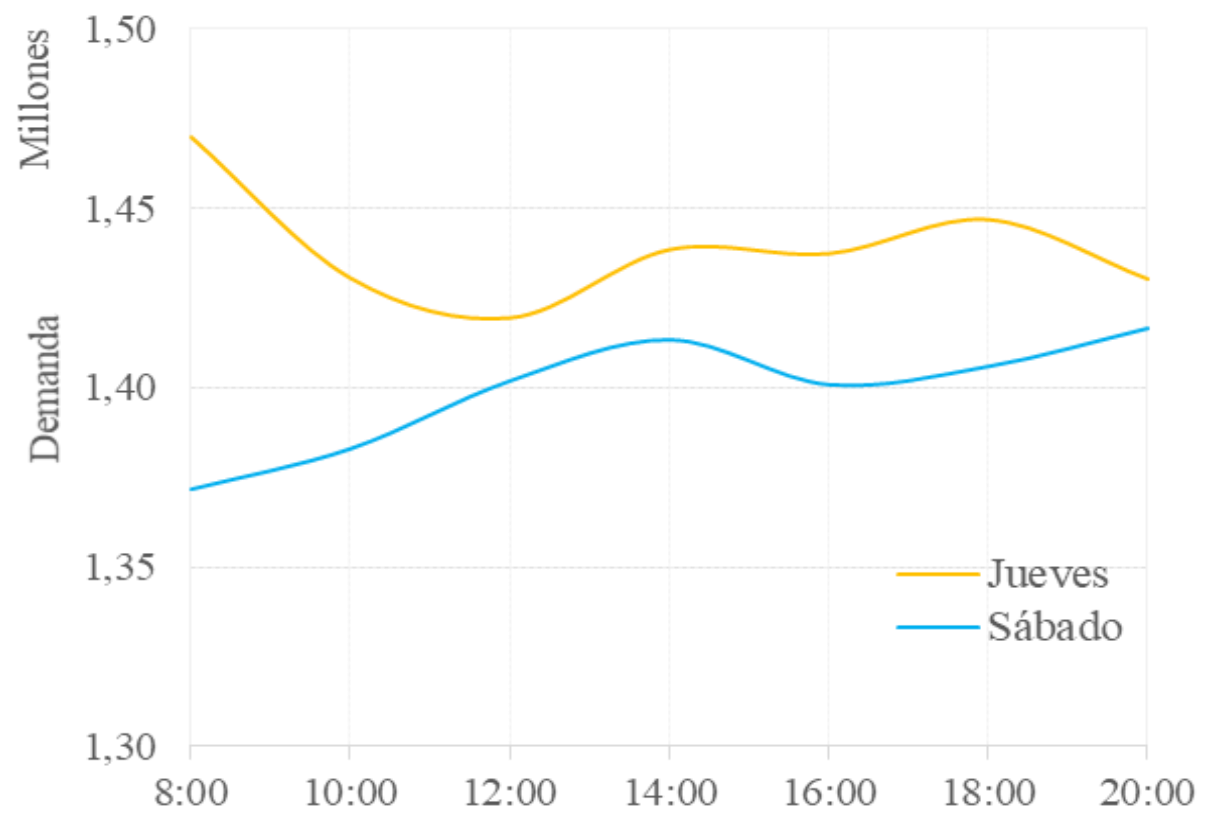

Figura 6. Demanda cubierta por hospital red GTFS.

Fte. Elaboración propia.

Tabla 2. Demanda cubierta por hospitales utilizando la red TomTom

\begin{tabular}{ccccccccr}
\hline \multirow{2}{*}{ Horas } & \multicolumn{3}{c}{ Jueves 30 minutos } & \multicolumn{4}{c}{ Sábado 30 minutos } \\
\cline { 2 - 9 } 08:00 & Total & $\%$ & Cambio & Cambio\% & Total & $\%$ & Cambio & Cambio\% \\
\cline { 2 - 9 } $10: 00$ & 2441791 & 75,8 & & & 2578802 & 80,0 & & \\
$12: 00$ & 2489449 & 77,2 & 47658 & 2,0 & 2567446 & 79,7 & -11356 & $-0,5$ \\
$14: 00$ & 2510085 & 77,2 & 45404 & 1,9 & 2554535 & 79,3 & -24267 & $-1,2$ \\
$16: 00$ & 2501574 & 77,6 & 68294 & 2,8 & 2561413 & 79,5 & -17389 & $-0,7$ \\
$18: 00$ & 2445007 & 75,9 & 3216 & 0,1 & 2548658 & 79,1 & -30144 & $-1,2$ \\
$20: 00$ & 2495596 & 77,4 & 53805 & 2,2 & 2548456 & 79,1 & -3346 & $-1,2$ \\
\hline
\end{tabular}

Fte. Elaboración propia.

Sí comparamos los resultados obtenidos por ambas redes, se nota que los cambios porcentuales son más altos en el transporte público que en el privado: el transporte público es más sensible a los cambios en las frecuencias que el coche a la congestión. Durante los sábados los perfiles son más estables, producto de una menor frecuencia del servicio de transporte y niveles de congestión más bajos en las calles. Además, el perfil cambia porque en coche desaparece la congestión de la mañana y de igual forma en el transporte público, por el contrario, la mejor accesibilidad se da al final de la mañana. En la Tabla 3 se observan las diferencias entre días laborables y los fines de semana donde la accesibilidad en coche es mejor el sábado que el jueves, al contrario que el transporte público (mejor jueves que sábado). 
Pérez Fernández, O. A. (2021). Modelos de localización asignación utilizando redes dinámicas a partir de ficheros GTFS y datos de TomTom. GeoFocus, Revista Internacional de Ciencia y Tecnología de la Información Geográfica (Artículos), 28, 59-81. http://dx.doi.org/10.21138/GF.751

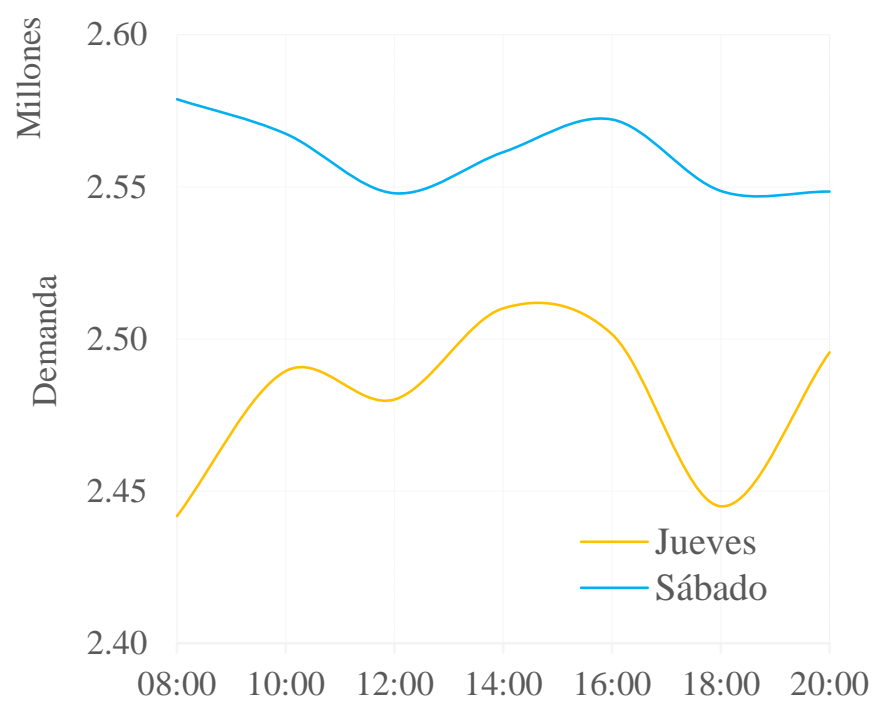

Figura 7. Demanda cubierta por hospital red TomTom.

Fte. Elaboración propia.

Tabla 3. Diferencias jueves sábado

\begin{tabular}{lrrrr}
\hline Horas & GTFS & \multicolumn{3}{c}{ TomTom } \\
& Total & $\%$ & Total & $\%$ \\
\hline 08:00 & -98107 & $-6,7$ & 137011 & 5,6 \\
10:00 & -47646 & $-3,3$ & 77997 & 3,1 \\
$12: 00$ & -17844 & $-1,3$ & 67340 & 2,7 \\
$14: 00$ & -25440 & $-1,8$ & 51328 & 2,0 \\
$16: 00$ & -36724 & $-2,6$ & 70567 & 2,8 \\
$18: 00$ & -41159 & $-2,8$ & 103651 & 4,2 \\
$20: 00$ & -14082 & $-1,0$ & 52860 & 2,1 \\
\hline
\end{tabular}

Fte. Elaboración propia.

La comparación del comportamiento de la demanda asignada a cada hospital para los dos días en estudio con ambas redes de transporte está plasmada en la Figura 8. Se apreció que los centros hospitalarios con mayor demanda asignada por el modelo, tanto jueves como sábado, son: el Hospital Central de la Defensa Gómez de Ulloa, Universitario de La Princesa, Universitario 12 de Octubre y Central de la Cruz Roja, con valores que oscilan entre los 70000 y 180000 habitantes. Analizando el porcentaje de cambio del sábado, en comparación con el jueves, se aprecia que la mayoría presentan porcentajes negativos que indican pérdida en los días de semana con relación a los fines de semana (Figura 8b).

Mientras que los resultados obtenidos de la red TomTom indican que muchos hospitales tienen demanda asignada superior durante los fines de semana (Figura 8c), como consecuencia de los menores niveles de congestión. Además, el porcentaje de cambio indicó una mayor cobertura los sábados (Figura 8d). 


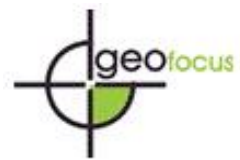

Pérez Fernández, O. A. (2021). Modelos de localización asignación utilizando redes dinámicas a partir de ficheros GTFS y datos de TomTom. GeoFocus, Revista Internacional de Ciencia y Tecnología de la Información Geográfica (Artículos), 28, 59-81. http://dx.doi.org/10.21138/GF.751

5.1 Modelos de localización con hospitales de referencia de las áreas de salud

La demanda cubierta por los hospitales de referencia asignados a las áreas de salud en que se divide el municipio de Madrid, utilizando la red de transporte público (GTFS), se resumen en la Tabla 4. De manera general, los datos reflejan que sólo los hospitales La Princesa y Santa Cristina (área de salud Centro Norte) cubren por encima del $100 \%$ de la población que vive dentro de los límites de su respetiva área de salud. Con el uso de la red TomTom, la mayoría de los hospitales no logran cubrir la población de su respectiva área de salud, excepto los hospitales La Princesa y 12 de Octubre (área de salud Sur II) que abarcan por encima del $100 \%$ de la demanda (Tabla 5).

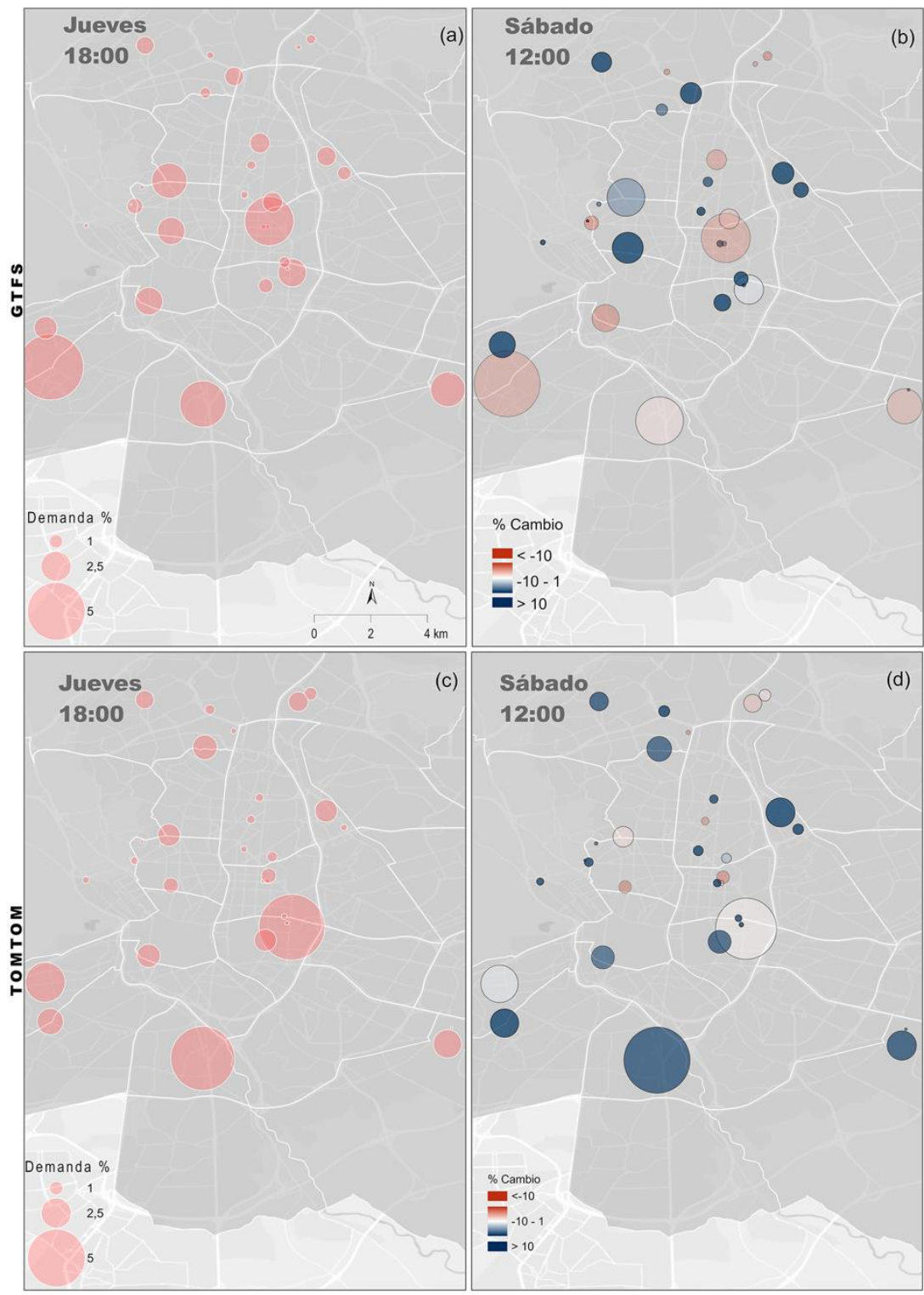

Figura 8. Demanda asignada y porcentaje de cambio.

Fte. Elaboración propia. 
Pérez Fernández, O. A. (2021). Modelos de localización asignación utilizando redes dinámicas a partir de ficheros GTFS y datos de TomTom. GeoFocus, Revista Internacional de Ciencia y Tecnología de la Información Geográfica (Artículos), 28, 59-81. http://dx.doi.org/10.21138/GF.751

Tabla 4. Demanda cubierta por los hospitales de las áreas de salud (jueves GTFS)

\begin{tabular}{|c|c|c|c|c|c|c|c|c|c|c|c|c|c|c|c|}
\hline \multirow[b]{2}{*}{ Hospital } & \multirow{2}{*}{$\begin{array}{l}\text { Población } \\
\text { dentro del } \\
\text { área de } \\
\text { salud }\end{array}$} & \multicolumn{2}{|c|}{ 08:00 } & \multicolumn{2}{|c|}{$10: 00$} & \multicolumn{2}{|c|}{$12: 00$} & \multicolumn{2}{|c|}{$14: 00$} & \multicolumn{2}{|c|}{$16: 00$} & \multicolumn{2}{|c|}{$18: 00$} & \multicolumn{2}{|c|}{ 20:00 } \\
\hline & & Total & $\%$ & Total & $\%$ & Total & $\%$ & Total & $\%$ & Total & $\%$ & Total & $\%$ & Total & $\%$ \\
\hline GMVT & 622597 & 134007 & 21,5 & 125840 & 20,2 & 119421 & 19,2 & 139531 & 22,4 & 139976 & 22,5 & 141660 & 22,8 & 127809 & 20,5 \\
\hline LPSC & 290181 & 428380 & 147,6 & 409624 & 141,2 & 411853 & 141,9 & 389450 & 134,2 & 420767 & 145,0 & 424986 & 146,5 & 419399 & 144,5 \\
\hline $\mathrm{RC}$ & 602501 & 27100 & 4,5 & 22868 & 3,8 & 19748 & 3,3 & 26582 & 4,4 & 22309 & 3,7 & 33917 & 5,6 & 30194 & 5,0 \\
\hline LP & 399128 & 138603 & 34,7 & 119468 & 29,9 & 127642 & 32,0 & 121314 & 30,4 & 115603 & 29,0 & 111044 & 27,8 & 124787 & 31,3 \\
\hline $\mathrm{PH}$ & 117873 & 3513 & 3,0 & 3865 & 3,3 & 3714 & 3,2 & 3720 & 3,2 & 3531 & 3,0 & 3742 & 3,2 & 3531 & 3,0 \\
\hline SCA & 506873 & 92000 & 18,2 & 93875 & 18,5 & 90876 & 17,9 & 90369 & 17,8 & 80064 & 15,8 & 83576 & 16,5 & 71824 & 14,2 \\
\hline 12OCT & 684181 & 196617 & 28,7 & 183635 & 26,8 & 168537 & 24,6 & 178080 & 26,0 & 200024 & 29,2 & 181024 & 26,5 & 201929 & 29,5 \\
\hline
\end{tabular}

GMVT (Gregorio Marañón y Virgen de La Torre) LPSC (La Princesa y Santa Cristina) RC (Ramón y Cajal) LP (La Paz) PH (Puerta de Hierro) SCA (San Carlos) 12OCT (12 de Octubre) Fte. Elaboración propia.

Tabla 5. Demanda cubierta por los hospitales de las áreas de salud (jueves TomTom)

\begin{tabular}{|c|c|c|c|c|c|c|c|c|c|c|c|c|c|c|c|}
\hline \multirow[b]{2}{*}{ Hospital } & \multirow{2}{*}{$\begin{array}{l}\text { Población } \\
\text { dentro del } \\
\text { área de } \\
\text { salud }\end{array}$} & \multicolumn{2}{|c|}{ 08:00 } & \multicolumn{2}{|c|}{$10: 00$} & \multicolumn{2}{|c|}{$12: 00$} & \multicolumn{2}{|c|}{$14: 00$} & \multicolumn{2}{|c|}{$16: 00$} & \multicolumn{2}{|c|}{$18: 00$} & \multicolumn{2}{|c|}{$20: 00$} \\
\hline & & Total & $\%$ & Total & $\%$ & Total & $\%$ & Total & $\%$ & Total & $\%$ & Total & $\%$ & Total & $\%$ \\
\hline GMVT & 622597 & 380208 & 61,1 & 391414 & 62,9 & 378937 & 60,9 & 413233 & 66,4 & 411098 & 66,0 & 401042 & 64,4 & 418310 & 67,2 \\
\hline LPSC & 290181 & 380542 & 131,1 & 422380 & 145,6 & 338670 & 116,7 & 381166 & 131,4 & 350244 & 120,7 & 313365 & 108,0 & 335573 & 115,6 \\
\hline $\mathrm{RC}$ & 602501 & 273476 & 45,4 & 196223 & 32,6 & 286004 & 47,5 & 284844 & 47,3 & 275198 & 45,7 & 271790 & 45,1 & 321415 & 53,3 \\
\hline LP & 399128 & 211497 & 53,0 & 181497 & 45,5 & 247293 & 62,0 & 217327 & 54,5 & 243831 & 61,1 & 247250 & 61,9 & 234888 & 58,9 \\
\hline $\mathrm{PH}$ & 117873 & 92964 & 78,9 & 45815 & 38,9 & 104746 & 88,9 & 101680 & 86,3 & 101348 & 86,0 & 103261 & 87,6 & 106955 & 90,7 \\
\hline SCA & 506873 & 135582 & 26,7 & 139473 & 27,5 & 141635 & 27,9 & 156586 & 30,9 & 157541 & 31,1 & 151842 & 30,0 & 163635 & 32,3 \\
\hline $12 \mathrm{O}$ & 684181 & 712622 & 104,2 & 753556 & 110,1 & 689247 & 100,7 & 667900 & 97,6 & 667189 & 97,5 & 641998 & 93,8 & 639458 & 93,5 \\
\hline
\end{tabular}

Fte. Elaboración propia. 


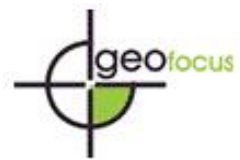

Pérez Fernández, O. A. (2021). Modelos de localización asignación utilizando redes dinámicas a partir de ficheros GTFS y datos de TomTom. GeoFocus, Revista Internacional de Ciencia y Tecnología de la Información Geográfica (Artículos), 28, 59-81. http://dx.doi.org/10.21138/GF.751

Por otro lado, con la ubicación de un nuevo hospital mejora la cobertura que brindan los hospitales actuales. Para el caso de la red GTFS el incremento oscila entre $17 \%$ y $23 \%$, mientras que con TomTom los valores rondan el $5 \%$ (Tabla 6 y 7). La Figura 9, muestra la ubicación del nuevo hospital y los hospitales actuales utilizando la red GTFS. Se observaron cambios en la ubicación del nuevo hospital en las horas 16:00 y 20:00. El color gris del círculo representa los hospitales de las áreas de salud actuales, mientras que el color chocolate muestra la ubicación del nuevo hospital. En todas las franjas horarias, el Hospital Universitario de La Princesa presentó los mejores niveles de cobertura, producto de un servicio de transporte con mayor eficiencia en esta zona. Además, el nuevo hospital abarca más demanda que la mayoría de los hospitales actuales. En el caso del modelo con la red TomTom, el nuevo hospital cambia de posición a las 8:00 y las 12:00 (Figura 10). Se observa que el Hospital Universitario 12 de Octubre registra la mayor demanda cubierta. Además, el nuevo hospital tiene una mayor cobertura que algunos de los hospitales existentes.

Tabla 6. Demanda cubierta por hospitales de referencia actuales y por el nuevo hospital (Red GTFS)

\begin{tabular}{|c|c|c|c|c|c|c|c|c|c|c|}
\hline \multirow{3}{*}{ Horas } & \multicolumn{5}{|c|}{ Jueves } & \multicolumn{5}{|c|}{ Sábado } \\
\hline & \multicolumn{2}{|c|}{$\begin{array}{c}\text { Hospitales } \\
\text { actuales }\end{array}$} & \multicolumn{3}{|c|}{$\begin{array}{c}\text { Con nuevo } \\
\text { Hospital }\end{array}$} & \multicolumn{2}{|c|}{$\begin{array}{c}\text { Hospitales } \\
\text { actuales }\end{array}$} & \multicolumn{3}{|c|}{$\begin{array}{c}\text { Con nuevo } \\
\text { hospital }\end{array}$} \\
\hline & Total & $\%$ & Total & $\%$ & Inc. $\%$ & Total & $\%$ & Total & $\%$ & Inc. $\%$ \\
\hline 08:00 & 1016852 & 31,5 & 1194455 & 37,1 & 17,5 & 867947 & 26,9 & 1064720 & 33,0 & 22,7 \\
\hline 10:00 & 955799 & 29,7 & 1143571 & 35,5 & 19,6 & 905577 & 28,1 & 1074488 & 33,3 & 18,7 \\
\hline $12: 00$ & 938328 & 29,1 & 1130135 & 35,1 & 20,4 & 905718 & 28,1 & 1111903 & 34,5 & 22,8 \\
\hline $14: 00$ & 945720 & 29,3 & 1140874 & 35,4 & 20,6 & 942585 & 29,2 & 1119795 & 34,7 & 18,8 \\
\hline $16: 00$ & 979203 & 30,4 & 1156255 & 35,9 & 18,1 & 914683 & 28,4 & 1097980 & 34,1 & 20,0 \\
\hline $18: 00$ & 976903 & 30,3 & 1166165 & 36,2 & 19,4 & 921602 & 28,6 & 1109648 & 34,4 & 20,4 \\
\hline 20:00 & 976169 & 30,3 & 1159040 & 36,0 & 18,7 & 944284 & 29,3 & 1123623 & 34,9 & 19,0 \\
\hline
\end{tabular}

Fte. Elaboración propia.

Tabla 7. Demanda cubierta por hospitales de referencia actuales y por el nuevo hospital (Red TomTom)

\begin{tabular}{|c|c|c|c|c|c|c|c|c|c|c|}
\hline \multirow{3}{*}{ Horas } & \multicolumn{5}{|c|}{ Jueves } & \multicolumn{5}{|c|}{ Sábado } \\
\hline & \multicolumn{2}{|c|}{$\begin{array}{c}\text { Hospitales } \\
\text { actuales }\end{array}$} & \multicolumn{3}{|c|}{$\begin{array}{c}\text { Con nuevo } \\
\text { hospital }\end{array}$} & \multicolumn{2}{|c|}{$\begin{array}{c}\text { Hospitales } \\
\text { actuales }\end{array}$} & \multicolumn{3}{|c|}{$\begin{array}{c}\text { Con nuevo } \\
\text { hospital }\end{array}$} \\
\hline & Total & $\%$ & Total & $\%$ & Inc.\% & Total & $\%$ & Total & $\%$ & Inc.\% \\
\hline $08: 00$ & 2128474 & 66,0 & 2231957 & 69,2 & 4,9 & 2334512 & 72,4 & 2414716 & 74,9 & 3,4 \\
\hline $10: 00$ & 2182031 & 67,7 & 2271915 & 70,5 & 4,1 & 2320608 & 72,0 & 2400964 & 74,5 & 3,5 \\
\hline $12: 00$ & 2180984 & 67,7 & 2272187 & 70,5 & 4,2 & 2304307 & 71,5 & 2384997 & 74,0 & 3,5 \\
\hline $14: 00$ & 2217936 & 68,8 & 2304962 & 71,5 & 3,9 & 2311864 & 71,7 & 2392607 & 74,2 & 3,5 \\
\hline $16: 00$ & 2201700 & 68,3 & 2290408 & 71,1 & 4,0 & 2325907 & 72,2 & 2406635 & 74,7 & 3,5 \\
\hline $18: 00$ & 2125254 & 65,9 & 2216679 & 68,8 & 4,3 & 2294923 & 71,2 & 2377281 & 73,8 & 3,6 \\
\hline $20: 00$ & 2214585 & 68,7 & 2299592 & 71,3 & 3,8 & 2293204 & 71,1 & 2375600 & 73,7 & 3,6 \\
\hline
\end{tabular}

Al comparar los mapas de las Figuras 9-10 es notable una mejor accesibilidad a los hospitales en coche privado, tal como muestran los tamaños de los círculos. La diferencia en la demanda captada por los hospitales que atienden las áreas de salud del municipio de Madrid está plasmada en la Tabla 8. En términos generales, se reflejó que con el uso de la red GTFS los jueves 


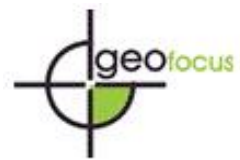

Pérez Fernández, O. A. (2021). Modelos de localización asignación utilizando redes dinámicas a partir de ficheros GTFS y datos de TomTom. GeoFocus, Revista Internacional de Ciencia y Tecnología de la Información Geográfica (Artículos), 28, 59-81. http://dx.doi.org/10.21138/GF.751

se captó mayor demanda que los sábados, ya que la mayoría de los hospitales presentaron cambios porcentuales negativos, excluyendo el Hospital Ramón y Cajal que registró valores positivos. Para el caso donde se utilizó la red TomTom ocurrió lo contrario, existe mayor demanda los sábados tal como lo indican los cambios porcentuales positivos (Tabla 9).

\section{Conclusiones}

En este artículo se propuso utilizar los datos GTFS y TomTom, para incorporar variables temporales sobre la red de transporte que se empleó en el planteamiento de los MLA. Se desarrollaron tres modelos de localización asignación. Primero, se propuso un modelo utilizando como candidatos toda la red de hospitales públicos del municipio de Madrid. En segundo lugar, se desarrolló otro modelo utilizando como candidatos los hospitales responsables de la atención a las áreas de salud. Por último, un modelo en el que se ensayó incorporar un nuevo hospital utilizando los actuales hospitales de referencia como ubicaciones requeridas, tomando como candidatos los centroides extraídos de las secciones censales. En los tres modelos, la demanda es obtenida a partir de los centroides de las secciones censales. Todos estos modelos han sido ejecutados en siete franjas horarias para los jueves y sábados.

El propósito de estos modelos consistió en evaluar el efecto de las frecuencias del transporte público y de los niveles de congestión, sobre la cobertura de la demanda a lo largo de diferentes franjas horarias. En previos trabajos de MLA, las redes utilizadas eran estáticas, solamente se contaba con datos de límites de velocidad y la longitud de los diferentes segmentos de calles. Resultaba difícil aplicar variables temporales como las frecuencias del servicio de transporte público, los niveles de congestión y la velocidad en diversas horas del día. Hoy gracias al surgimiento de las nuevas fuentes de datos como, los ficheros GTFS (General Transit Feed Specification) y los perfiles de velocidad de TomTom, es posible alimentar la red de calles con atributos que permiten hacer un acercamiento más ajustado a la movilidad real de la demanda.

Los resultados de esta investigación demuestran que la cantidad de demanda a captar por un servicio puede aumentar o disminuir, dependiendo de la variación en las frecuencias del transporte público o de las condiciones del tráfico de las calles en las diversas franjas horarias del día. El modelo consideró estudiar el efecto en la demanda cubierta por los hospitales en algunas franjas horarias de dos días de la semana, con datos del transporte público (GTFS) y el transporte privado (TomTom), descubriendo que en ciertas horas pico la demanda que atienden los hospitales es afectada, de acuerdo con las condiciones de la red de calles. En el caso de la MLA con todos los hospitales, los resultados indican una mejor cobertura de la demanda los jueves en comparación con los sábados, este comportamiento obedece a la menor frecuencia del transporte público los fines de semana. Por otro lado, lo contrario ocurre con la red TomTom, con una mejor cobertura los sábados que los jueves, a causa de los menores niveles de congestión de los fines de semana. En particular, los resultados del modelo realizado con los hospitales de referencia de las áreas de salud sugieren que en ambas redes de transporte solo aparece un hospital que consigue cubrir más del $100 \%$ de la población del área de salud, observándose una mejor cobertura en los modelos realizados con la red TomTom. Finalmente, los resultados del modelo que incorpora un nuevo hospital indican una mejora en los porcentajes de cobertura, llegando a superar el $50 \%$ en el caso de la red TomTom. 
Pérez Fernández, O. A. (2021). Modelos de localización asignación utilizando redes dinámicas a partir de ficheros GTFS y datos de TomTom. GeoFocus, Revista Internacional de Ciencia y Tecnología de la Información Geográfica (Artículos), 28, 59-81. http://dx.doi.org/10.21138/GF.751

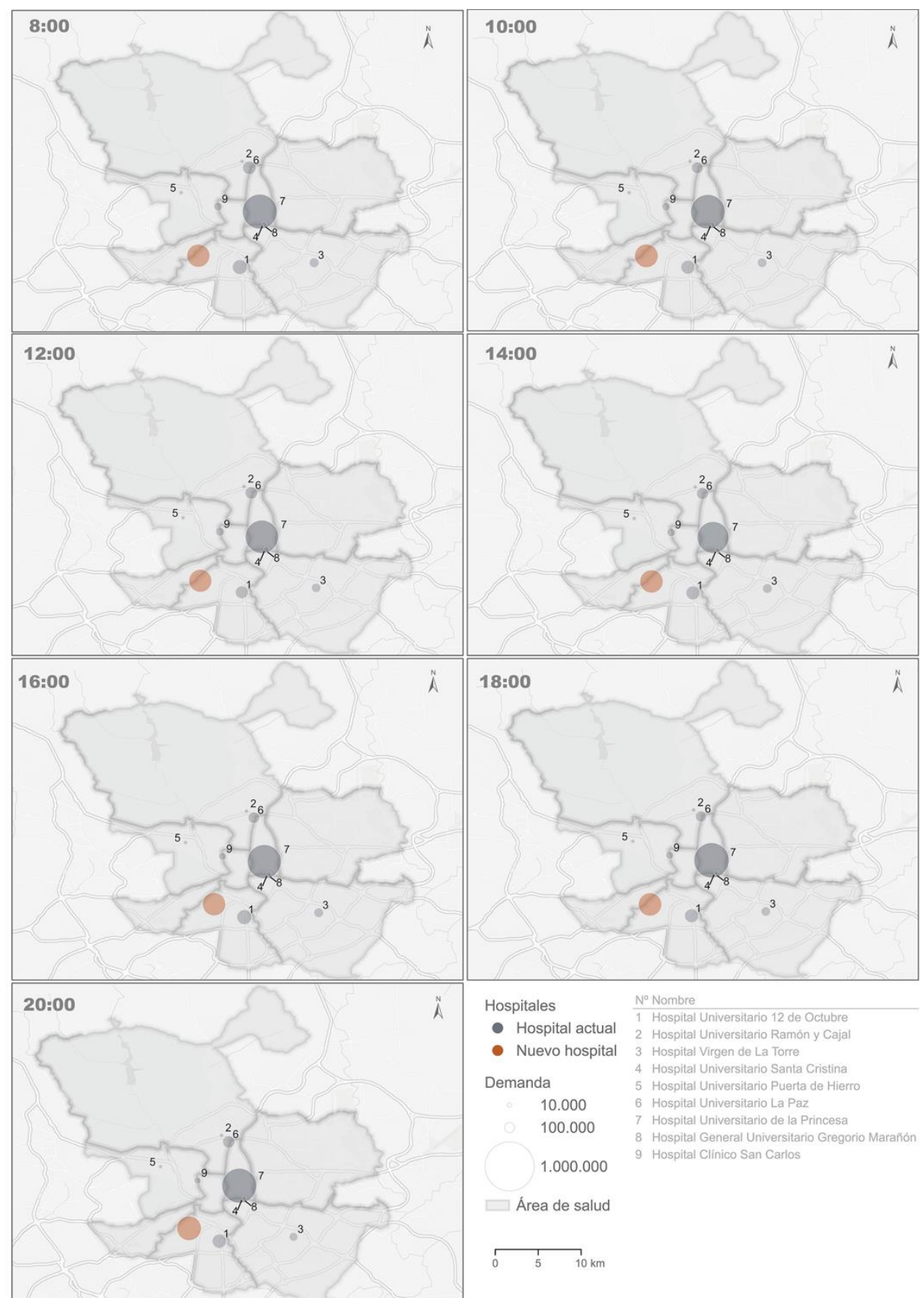

Figura 9. Demanda cubierta por los hospitales de referencia y el nuevo hospital (jueves red GTFS).

Fte. Elaboración propia. 
Pérez Fernández, O. A. (2021). Modelos de localización asignación utilizando redes dinámicas a partir de ficheros GTFS y datos de TomTom. GeoFocus, Revista Internacional de Ciencia y Tecnología de la Información Geográfica (Artículos), 28, 59-81. http://dx.doi.org/10.21138/GF.751

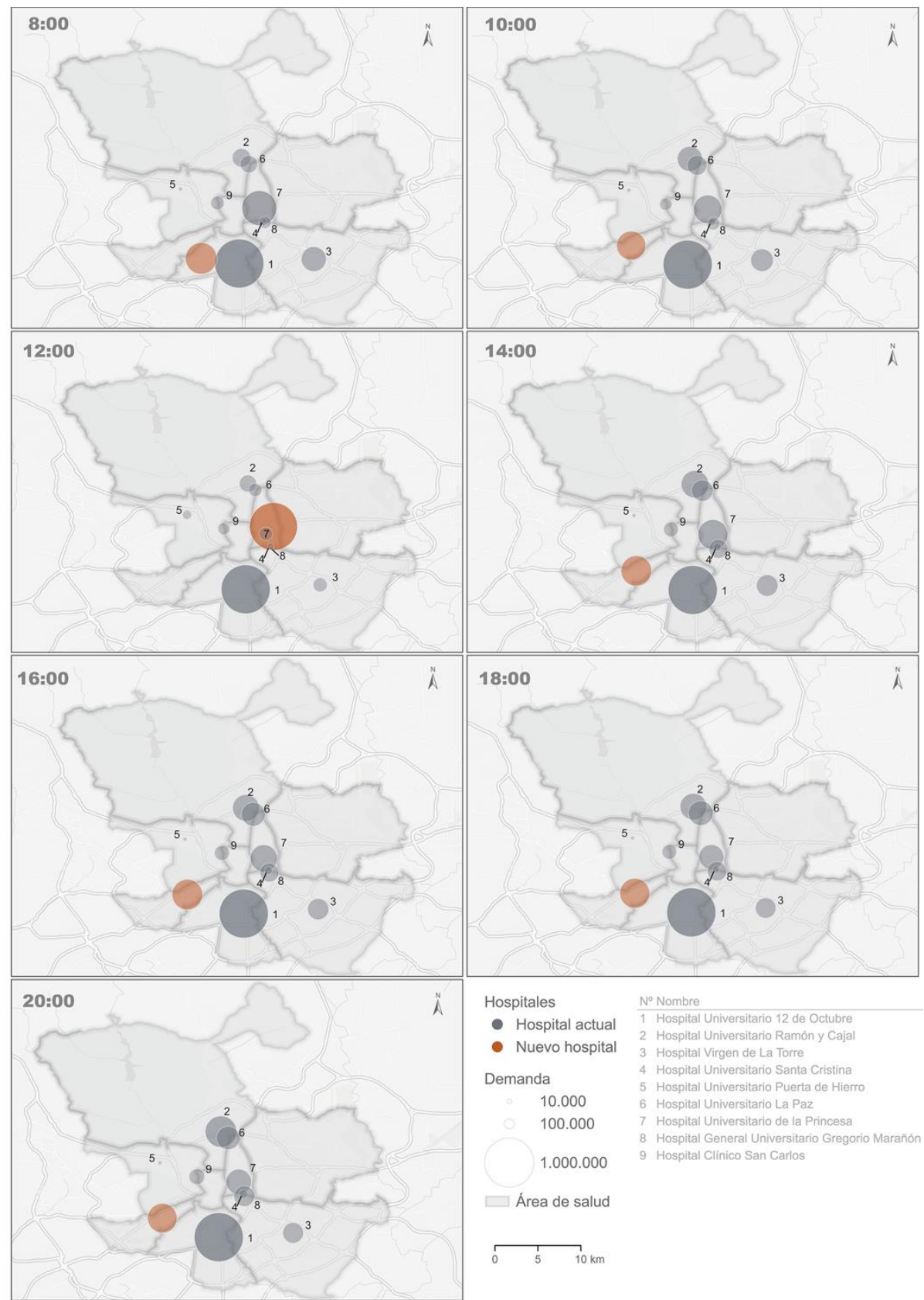

Figura 10. Demanda cubierta por los hospitales de referencia y el nuevo hospital (jueves red TomTom)

Fte. Elaboración propia. 
Pérez Fernández, O. A. (2021). Modelos de localización asignación utilizando redes dinámicas a partir de ficheros GTFS y datos de TomTom. GeoFocus, Revista Internacional de Ciencia y Tecnología de la Información Geográfica (Artículos), 28, 59-81. http://dx.doi.org/10.21138/GF.751

Tabla 8. Diferencia jueves y sábado hospitales de referencia red GTFS

\begin{tabular}{|c|c|c|c|c|c|c|c|c|c|c|c|c|c|c|}
\hline \multirow[t]{2}{*}{ Hospital } & \multicolumn{2}{|c|}{ 08:00 } & \multicolumn{2}{|c|}{$10: 00$} & \multicolumn{2}{|c|}{$12: 00$} & \multicolumn{2}{|c|}{$14: 00$} & \multicolumn{2}{|c|}{$16: 00$} & \multicolumn{2}{|c|}{ 18:00 } & \multicolumn{2}{|c|}{$20: 00$} \\
\hline & Total & $\%$ & Total & $\%$ & Total & $\%$ & Total & $\%$ & Total & $\%$ & Total & $\%$ & Total & $\%$ \\
\hline HGMVT & -8959 & $-6,7$ & 2343 & 1,9 & 13941 & 11,7 & -18850 & $-13,5$ & -14591 & $-10,4$ & -2524 & $-1,8$ & -4417 & $-3,5$ \\
\hline HLPSC & -48091 & $-11,2$ & -15536 & $-3,8$ & -28398 & $-6,9$ & -10270 & $-2,6$ & -20729 & $-4,9$ & -42888 & $-10,1$ & -54940 & $-13,1$ \\
\hline HRC & -10373 & $-38,3$ & 6726 & 29,4 & 6977 & 35,3 & -1337 & $-5,0$ & 600 & 2,7 & 19215 & 56,7 & 3271 & 10,8 \\
\hline HLP & -17836 & $-12,9$ & -11224 & $-9,4$ & -13415 & $-10,5$ & -6815 & $-5,6$ & -9235 & $-8,0$ & -13416 & $-12,1$ & -8383 & $-6,7$ \\
\hline $\mathrm{HPH}$ & -393 & $-11,2$ & -788 & $-20,4$ & 534 & 14,4 & -554 & $-14,9$ & -365 & $-10,3$ & -665 & $-17,8$ & -482 & $-13,7$ \\
\hline HSCA & -33663 & $-36,6$ & 19323 & 20,6 & -6933 & $-7,6$ & -4840 & $-5,4$ & 2737 & 3,4 & -4779 & $-5,7$ & 19266 & 26,8 \\
\hline $\mathrm{H} 12 \mathrm{O}$ & -29913 & $-15,2$ & 12867 & 7,0 & -4532 & $-2,7$ & 39243 & 22,0 & -22861 & $-11,4$ & -10649 & $-5,9$ & 13544 & 6,7 \\
\hline
\end{tabular}

Tabla 9. Diferencia jueves y sábado hospitales de referencia red TomTom

\begin{tabular}{|c|c|c|c|c|c|c|c|c|c|c|c|c|c|c|}
\hline \multirow{2}{*}{ Hospital } & \multicolumn{2}{|c|}{ 08:00 } & \multicolumn{2}{|c|}{$10: 00$} & \multicolumn{2}{|c|}{$12: 00$} & \multicolumn{2}{|c|}{$14: 00$} & \multicolumn{2}{|c|}{$16: 00$} & \multicolumn{2}{|l|}{$18: 00$} & \multicolumn{2}{|c|}{$20: 00$} \\
\hline & Total & $\%$ & Total & $\%$ & Total & $\%$ & Total & $\%$ & Total & $\%$ & Total & $\%$ & Total & $\%$ \\
\hline HGMVT & 66827 & 17,6 & 53657 & 13,7 & 60701 & 16,0 & 28467 & 6,9 & 34744 & 8,5 & 36809 & 9,2 & 6217 & 1,5 \\
\hline HLPSC & 77701 & 20,4 & 31105 & 7,4 & 109071 & 32,2 & 59865 & 15,7 & 95422 & 27,2 & 123538 & 39,4 & 104130 & 31,0 \\
\hline HRC & 64689 & 23,7 & 145631 & 74,2 & 68332 & 23,9 & 59461 & 20,9 & 68759 & 25,0 & 74545 & 27,4 & -11934 & $-3,7$ \\
\hline HLP & -52689 & $-24,9$ & -28030 & $-15,4$ & -103434 & $-41,8$ & -56794 & $-26,1$ & -85377 & $-35,0$ & -92494 & $-37,4$ & -42440 & $-18,1$ \\
\hline $\mathrm{HPH}$ & 15845 & 17,0 & 62933 & 137,4 & 9684 & 9,2 & 14413 & 14,2 & 7141 & 7,0 & 10648 & 10,3 & 10102 & 9,4 \\
\hline HSCA & 27355 & 20,2 & 22708 & 16,3 & 14623 & 10,3 & 2337 & 1,5 & 5931 & 3,8 & 6954 & 4,6 & -8258 & $-5,0$ \\
\hline $\mathrm{H} 12 \mathrm{O}$ & -45607 & $-6,4$ & -91107 & $-12,1$ & -34329 & $-5,0$ & -11900 & $-1,8$ & -662 & $-0,1$ & 11560 & 1,8 & 22463 & 3,5 \\
\hline
\end{tabular}

Fte. Elaboración propia. 
Pérez Fernández, O. A. (2021). Modelos de localización-asignación utilizando redes dinámicas a partir de ficheros GTFS y datos de TomTom. GeoFocus, Revista Internacional de Ciencia y Tecnología de la Información Geográfica, 28, 35-57. http://dx.doi.org/10.21138/GF.751

Esta investigación tiene varias aplicaciones prácticas: en primer lugar, las instalaciones de servicio pueden ajustar su nivel de atención, por ejemplo: dosificando la mano de obra, tomando como referencia las horas donde existe mayor cobertura de la demanda. En segundo lugar, modificar los horarios de apertura o cierre de los negocios o equipamientos. A diferencia de estudios previos, donde los resultados obtenidos solo eran representativos de un momento fijo, con la incorporación de ficheros GTFS y datos de TomTom es posible analizar la variabilidad por horas, días o el periodo temporal que sea de interés.

En investigaciones futuras, se podrá incorporar otras fuentes de Big Data para obtener la demanda y la oferta de servicios, considerando la variabilidad en el espacio y tiempo. Hay abundante terreno para seguir avanzando en esta temática, se pueden incluir redes dinámicas como las que aporta Waze, Google Maps y Here, para evaluar las condiciones del tráfico en tiempo real. Cabe la posibilidad de aplicar estos modelos en otras ciudades y para cualquier tipo de servicio, así como utilizar otras variables de investigación al plantear modelos de localización que incluyan el dinamismo en sus tres principales componentes, evaluando los cambios de la demanda, oferta y la red en las diferentes horas del día.

\section{Referencias bibliográficas}

Ajebon, M. O., y Asikhia, M. O. (2013): "Geographical analysis of smoking in Leeds: A GISbased location-allocation technique for the optimal location of smoking cessation services", Journal of Geographic Information System, 05(June), 269-280. https://doi.org/10.4236/jgis.2013.53026

Alonso, A. B. (2016): "Optimal location of solid waste containers in Alcala de Henares", Revista Electronica De Medio Ambiente, 17(1), 1-23. https://doi.org/10.5209/MARE.53155

Antrim, A., y Barbeau, S. J. (2013): "The many uses of gtfs data - opening the door transit and multimodal application", Location-aware information systems laboratory at the University of South Florida, 1-24. https://doi.org/10.1.1.391.5421

Brandeau, M., y Chiu, S. S. (1989): "An overview of representative problems in location research", Management Science, 35(6), 645-674. https://doi.org/10.1287/mnsc.35.6.645

Chukwusa, E., y Comber, A. (2018). "The impact of residential and non-residential demand on location-allocation decision-making: A case study of modelling suitable locations for ems in Leicester and Leicestershire, England UK", Journal of Geographic Information System, 10(04), 381-397. https://doi.org/10.4236/jgis.2018.104020

Dingil, A. E., Schweizer, J., Rupi, F., y Stasiskiene, Z. (2018): "Transport indicator analysis and comparison of 151 urban areas, based on open source data", European Transport Research Review. https://doi.org/10.1186/s12544-018-0334-4

El-Hallaq, M. A., y Mosabeh, R. (2019): "Optimization of municipal solid waste management of bins using gis. A case study: Nuseirat city", Journal of Geographic Information System, 11(01), 32-43. https://doi.org/10.4236/jgis.2019.111003

Erfani, S. M., Danesh, S., Karrabi, S. M., Shad, R., y Nemati, S. (2018): "Using applied operations research and geographical information systems to evaluate effective factors in storage service of municipal solid waste management systems", Waste Management, 79, 346-355. https://doi.org/10.1016/j.wasman.2018.08.003 


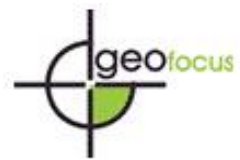

Pérez Fernández, O. A. (2021). Modelos de localización-asignación utilizando redes dinámicas a partir de ficheros GTFS y datos de TomTom. GeoFocus, Revista Internacional de Ciencia y Tecnología de la Información Geográfica, 28, 35-57. http://dx.doi.org/10.21138/GF.751

Farber, S., Morang, M. Z., y Widener, M. J. (2014): "Temporal variability in transit-based accessibility to supermarkets", Applied Geography, 53, 149-159. https://doi.org/10.1016/j.apgeog.2014.06.012

Fayyaz S., Liu, X. C., y Zhang, G. (2017): "An efficient general transit feed specification (GTFS) enabled algorithm for dynamic transit accessibility analysis", PLOS ONE, 12(10), 1-22. https://doi.org/10.1371/journal.pone.0185333

García-Palomares, J. C., Gutiérrez, J. , y Latorre, M. (2012): "Optimizing the location of stations in bike-sharing programs: A GIS approach", Applied Geography, 35(1-2), 235-246. https://doi.org/10.1016/j.apgeog.2012.07.002

García-Palomares, J. C., Salas-Olmedo, M. H., Moya-Gómez, B., Condeço-Melhorado, A., y Gutiérrez, J. (2018): "City dynamics through Twitter: Relationships between land use and spatiotemporal demographics", Cities, 72(June 2017), 310-319. https://doi.org/10.1016/j.cities.2017.09.007

Instituto Nacional de Estadística de España. (2020, octubre 1). Padrón de población. https://www.ine.es/

Jakimavičius, M., Palevičius, V., Antuchevičiene, J., y Karpavičius, T. (2019): "Internet gis-based multimodal public transport trip planning information system for travelers in Lithuania", ISPRS International Journal of Geo-Information, 8(8), 319. https://doi.org/10.3390/ijgi8080319

Karner, A. (2018): "Assessing public transit service equity using route-level accessibility measures and public data", Journal of Transport Geography, 67(June 2017), 24-32. https://doi.org/10.1016/j.jtrangeo.2018.01.005

Kotavaara, O. (2018): "Integrated location-allocation of private car and public transport users primary health care facility allocation in the Oulu Region of Finland", 21st International Conference on Geographic Information Science (AGILE 2018), 1-8. http://jultika.oulu.fi/Record/nbnfi-fe2019052116475

Moya-Gómez, B., y García-Palomares, J. C. (2017): "The daily dynamic potential accessibility by car in London on wednesdays", Journal of Maps, 13(1), 31-39. https://doi.org/10.1080/17445647.2017.1305301

Moya-Gómez, B., Henar Salas-Olmedo, M., Carlos García-Palomares, J., y Gutiérrez, J. (2018): "Dynamic accessibility using big data: The role of the changing conditions of network congestion and destination attractiveness" Springer Science+Business Media. https://doi.org/10.1007/s11067-017-9348-z

Moyano, A., Moya-Gómez, B., y Gutiérrez, J. (2018): "Access and egress times to high-speed rail stations: a spatiotemporal accessibility analysis", Journal of Transport Geography, 73(March), 84-93. https://doi.org/10.1016/j.jtrangeo.2018.10.010

Nassir, N., Khani, A., Lee, S. G., Noh, H., y Hickman, M. (2012): "Transit stop-level origindestination estimation through use of transit schedule and automated data collection system", Transportation Research Record: Journal of the Transportation Research Board, 2263(1), 140150. https://doi.org/10.3141/2263-16

Özceylan, E., Uslu, A., Erbaş, M., Çetinkaya, C., y Kürşat, S. (2017): "Optimizing the locationallocation problem of pharmacy warehouses: A case study in Gaziantep", An International Journal of Optimization and Control: Theories \& Applications, 7(1), 117-129. https://doi.org/10.11121/ijocta.01.2017.00373 
Pérez Fernández, O. A. (2021). Modelos de localización-asignación utilizando redes dinámicas a partir de ficheros GTFS y datos de TomTom. GeoFocus, Revista Internacional de Ciencia y Tecnología de la Información Geográfica, 28, 35-57. http://dx.doi.org/10.21138/GF.751

Park, C., y Young, S. (2017): "An optimization approach for the placement of bicycle-sharing stations to reduce short car trips: an application to the city of Seoul", Transportation Research Part A: Policy and Practice, 105(June), 154-166. https://doi.org/10.1016/j.tra.2017.08.019

Pritchard, J. P., Tomasiello, D. B., Giannotti, M., y Geurs, K. (2019): "Potential impacts of bikeand-ride on job accessibility and spatial equity in São Paulo, Brazil", Transportation Research Part A: Policy and Practice, 121(May 2018), 386-400. https://doi.org/10.1016/j.tra.2019.01.022

Rahman, M., Chen, N., Islam, M. M., Dewan, A., Pourghasemi, H. R., Washakh, R. M. A., Nepal, N., Tian, S., Faiz, H., Alam, M., y Ahmed, N. (2021): "Location-allocation modeling for emergency evacuation planning with GIS and remote sensing: A case study of Northeast Bangladesh", Geoscience Frontiers, 12(3), 101095. https://doi.org/10.1016/j.gsf.2020.09.022

Slovic, A. D., Tomasiello, D. B., Giannotti, M., Andrade, M. de F., y Nardocci, A. C. (2019): "The long road to achieving equity: Job accessibility restrictions and overlapping inequalities in the city of São Paulo", Journal of Transport Geography, 78(November 2018), 181-193. https://doi.org/10.1016/j.jtrangeo.2019.06.003

Stępniak, M., y Goliszek, S. (2017): "Spatio-temporal variation of accessibility by public transport-the equity perspective", Lecture Notes in Geoinformation and Cartography, 241-261. https://doi.org/10.1007/978-3-319-45123-7_18

Stępniak, M., Pritchard, J. P., Geurs, K. T., y Goliszek, S. (2019): "The impact of temporal resolution on public transport accessibility measurement: Review and case study in Poland", Journal of Transport Geography, 75, 8-24. https://doi.org/10.1016/j.jtrangeo.2019.01.007

Tanveer, H., Balz, T., Cigna, F., y Tapete, D. (2020): "Monitoring 2011-2020 traffic patterns in Wuhan (China) with COSMO-SkyMed SAR", Remote Sensing, 12(10). https://doi.org/10.3390/rs12101636

Tu, W., Li, Q., Fang, Z., Shaw, S. L., Zhou, B., y Chang, X. (2016): "Optimizing the locations of electric taxi charging stations: A spatial-temporal demand coverage approach", Transportation Research Part C: Emerging Technologies, 65(3688), 172-189. https://doi.org/10.1016/j.trc.2015.10.004

Wondwossen, M., y Bedasa, A. (2019). "Location allocation analysis for urban public services using gis techniques : A case of primary schools", American Journal of Geographic Information System, 8(1), 26-38. https://doi.org/10.5923/j.ajgis.20190801.03

Yang, L., Zhang, F., Kwan, M., Wang, K., Zuo, Z., y Xia, S. (2020): "Space-time demand cube for spatial-temporal coverage optimization model of shared bicycle system : A study using big bike GPS data", Journal of Transport Geography, 88(September 2019), 102861. https://doi.org/10.1016/j.jtrangeo.2020.102861

Zhang, Y., Lin, D., y Mi, Z. (2019): "Electric fence planning for dockless bike-sharing services", Journal of Cleaner Production, 206, 383-393. https://doi.org/10.1016/j.jclepro.2018.09.215

Zhou, L., Wang, S., y Xu, Z. (2020): "A multi-factor spatial optimization approach for emergency medical facilities in Beijing", International Journal of Geo-Information, 9(6). https://doi.org/10.3390/ijgi9060361. 
